\title{
COMPACT-COVERING IMAGES OF METRIC SPACES
}

\author{
E. MICHAEL ${ }^{1}$ AND K. NAGAMI
}

ABSTRACT. Several kinds of compact-covering images of metric spaces are characterized.

1. Introduction. A continuous map $f: X \rightarrow Y$ is called compact-covering if every compact subset of $Y$ is the image of some compact subset of $X$. This concept has been studied by N. Bourbaki [6, $\$ 1$, Proposition 18], A. V. Arhangel'skiǐ [2], E. Michael ([15, Corollary 1.2(b)], [17, Corollary 1.2], [18]), K. Nagami [21], and others. Compact-covering images of separable metric spaces were characterized in [18, Theorem 11.4]. The purpose of this paper is to characterize three kinds of compact-covering images of arbitrary metric spaces.

All maps in this paper are continuous, and all spaces are Hausdorff.

THEOREM 1.1. A space $Y$ is a compact-covering image of a metric space if and only if every compact subset of $Y$ is metrizable. ${ }^{2}$

THEOREM 1.2. A space $Y$ is a compact-covering open image of a metric space if and only if every compact subset of $Y$ is metrizable and of countable character $^{3}$ in $Y$.

THEOREM 1.3. A space $Y$ is a compact-covering open s-image $e^{4}$ of a metric space if and only if it has a point-countable base.

The condition in Theorem 1.1 is satisfied, for example, if $Y$ has a $G_{\delta}$-diagonal, since every compact Hausdorff space with a $G_{\delta}$-diagonal is metrizable.

Presented to the Society, January 18, 1972; received by the editors January 24, 1972. AMS (MOS) subject classifications (1969). Primary 5420, 5460; Secondary 5435, 5452.

Key words and phrases. Compact-covering map, metric space, open map, s-map, point-countable base, countable character.

${ }^{1}$ Partly supported by an N.S.F. grant.

2 This result was obtained independently by $\mathrm{F}$. Siwiec.

${ }^{3}$ A set $K \subset Y$ is of countable character in $Y$ if there is a countable outer base $\left(U_{n}\right)$ at $K$ in $Y$ (i.e. each $U_{n}$ is open and contains $K$, and every open set containing $K$ contains some $U_{n}$ ).

4 A map $f: X \rightarrow Y$ is an s-map if every $f^{-1}(y)$ is separable.

(c) American Mathematical Society 1973 
The condition in Theorem 1.2 is satisfied, for example, if $Y$ is developable. ${ }^{5}$ This can be inferred from a remark by R. W. Heath in $[13$, p. 106], and is easily verified using Lemma 3.1 .

It should be observed that Theorem 1.3 can be combined with known results to obtain the following theorem. (The equivalence of (a) and (c) was established by V. I. Ponomarev [22], while the fact that (d) implies (a) was proved by V. V. Filippov [10, Theorem 1.1].)

THEOREM 1.4. The following properties of a space $Y$ are equivalent.

(a) $Y$ has a point-countable base.

(b) $Y$ is a compact-covering open s-image of a metric space.

(c) $Y$ is an open s-image of a metric space.

(d) $Y$ is a bi-quotient s-image of a metric space.

Let us conclude this introduction with a question. Observe that, by Theorem 1.4, every open s-image of a metric space is also a compactcovering open $s$-image of a metric space (under a different map, in general). Analogously, it was proved in [18, Theorem 11.4 and Corollary 11.5] that every quotient image of a separable metric space is also a compactcovering quotient image of a separable metric space. We now ask:

Problem 1.5. Is every quotient $s$-image of a metric space also a compact-covering quotient $s$-image of a metric space?

For a positive solution to Problem 1.5, it would suffice to show that every quotient $s$-image $Y$ of a metric space is also a compact-covering $s$-image of some metric space. (That $Y$ must be a compact-covering image-rather than s-image-of some metric space follows from Theorem 1.1 and $[10$, Corollary 3$]$.)

The simple proof of Theorem 1.1 is given in $\$ 2$. Section 3 contains some lemmas needed in the proofs of Theorems 1.2 and 1.3, and $\S 4$ contains the proofs of those theorems. Section 5 is devoted to examples.

2. Proof of Theorem 1.1. The necessity of the condition is clear, since every Hausdorff continuous image of a compact metric space is metrizable. To prove sufficiency, suppose that every compact subset of $Y$ is metrizable. Let $X$ be the disjoint union (=topological sum) of all the compact subsets of $Y$, and let $f: X \rightarrow Y$ be the obvious map. Then $X$ is metrizable and $f$ is compact-covering, so that completes the proof.

3. Some lemmas. If $A \subset Y$, then a $Y$-base for $A$ is a collection $\mathscr{U}$ of open subsets of $Y$ such that, if $y \in A$ and $V$ is a neighborhood of $y$ in $Y$,

${ }^{5}$ A space $Y$ is developable if it has a development, i.e. a sequence of open covers $\mathscr{G}_{n}$ such that, if $y \in Y$, then $\left\{\operatorname{St}\left(y, \mathscr{G}_{n}\right): n \in N\right\}$ is a base at $y$ in $Y$. 
then $y \in U \subset V$ for some $U \in \mathscr{U} .^{6}$ The harder part of the following lemma $((a) \rightarrow(b))$ was proved by M. M. Coban in [7, Theorem 1]; for completely regular $Y$, it had previously been obtained by A. V. Arhangel'skii [3, Chapter III, Lemma 3.2].

LEMMA 3.1. The following properties of a compact subset $K$ of a space $Y$ are equivalent.

(a) $K$ is metrizable and of countable character in $Y$.

(b) K has a countable Y-base.

Proof. (a) $\rightarrow($ b). As already observed, this was proved by Coban [7, Theorem 1].

(b) $\rightarrow$ (a). Let $\mathscr{U}$ be a countable $Y$-base for $K$. Then $K$ has a countable base, and is thus metrizable. Moreover, if $\left\{\mathscr{U}_{n}: n \in N\right\}$ is the family of finite subcollections of $\mathscr{U}$ which cover $K$, then $\left\{\bigcup \mathscr{U}_{n}: n \in N\right\}$ is easily seen to be a countable outer base at $K$ in $Y$.

LEMMA 3.2. If $Y$ has a point-countable base $\mathscr{U}$, and if $K \subset Y$ is compact, then $K$ has a countable $Y$-base $\mathscr{V} \subset \mathscr{U}$ :

Proof. By a result of A. S. Miščenko [20, Theorem 1], $K$ intersects only countably many elements of $\mathscr{U}$, and these clearly form a countable $Y$-base for $K$.

LEMmA 3.3. Suppose that $K \subset Y$ is compact and has a countable $Y$-base $\mathscr{U}$. Then there is a sequence $\left(\mathscr{U}_{n}\right)$ of finite subcollections of $\mathscr{U}$ which cover $K$ such that:

(a) If $y \in K$, and if $y \in U_{n} \in \mathscr{U}_{n}$ for all $n$, then $\left(U_{n}\right)$ is a base at $y$ in $Y$.

(b) If $y \in K$, then there exist $U_{n} \in \mathscr{U}_{n}$ such that $y \in U_{n}$ and $\left(U_{n+1} \cap K\right)^{-\subset}$ $U_{n}$ for all $n$.

Proof. Let $\left(\mathscr{V}_{n}\right)$ be all the finite subcollections of $\mathscr{U}$ which cover $K$, arranged in a sequence. By induction, choose finite $\mathscr{U}_{n} \subset \mathscr{U}$ covering $K$ so that $\mathscr{U}_{n}$ refines $\mathscr{V}_{n}$ and $\left\{(U \cap K)^{-}: U \in \mathscr{U}_{n+1}\right\}$ refines $\mathscr{U}_{n}$ for all $n$. Let us check (a) and (b).

(a) Suppose that $y \in W$ with $W$ open in $Y$, and let us show that $U_{n} \subset W$ for some $n$. Pick $V \in \mathscr{U}$ such that $y \in V \subset W$, and then pick a finite subcollection $\mathscr{F}$ of $\mathscr{U}$ such that $K-V \subset \cup \mathscr{F}$ and $y \notin \bigcup \mathscr{F}$. Then $\mathscr{F} \cup\{V\}$ covers $K$, so $\mathscr{F} \cup\{V\}=\mathscr{V}_{n}$ for some $n$. Clearly $V$ is the only element of $\mathscr{V}_{n}$ containing $y$. Since $y \in U_{n}$, it follows that $U_{n} \subset V \subset W$.

(b) For each $n$, let $\mathscr{U}_{n}(y)=\left\{U \in \mathscr{U}_{n}: y \in U\right\}$. Clearly each $\mathscr{U}_{n}(y)$ is finite, and if $U \in \mathscr{U}_{n+1}(y)$ then $(U \cap K)^{-} \subset U^{\prime}$ for some $U^{\prime} \in \mathscr{U}_{n}(y)$. By

\footnotetext{
${ }^{6}$ This concept was called an exterior base for $A$ in $Y$ by A. V. Arhangel'skii [1, Definition 2(b)]. It should not be confused with what we call an outer base at $A$ in $Y$ (see footnote 3).
} 
König's infinity lemma (see, for instance, [16, Lemma 1]), one can choose $U_{n} \in \mathscr{U}_{n}(y)$ so that $\left(U_{n+1} \cap K\right)-\subset U_{n}$ for all $n$. That completes the proof.

4. Proofs of Theorems 1.2 and 1.3. The necessity of the conditions in these theorems can be easily checked by the reader, remembering that every metric space has a $\sigma$-locally finite base. The sufficiency follows from the following theorem, together with Lemmas 3.1 and 3.2.

THEOREM 4.1. Let $Y$ be first-countable, and let $\mathscr{U}$ be a base for $Y$. Then there exists a metric space $X$, and an open map $f: X \rightarrow Y$, with the following properties:

(a) If $K \subset Y$ is compact and has a countable $Y$-base $\mathscr{U}(K) \subset \mathscr{U}$, then $K=f(C)$ for some compact $C \subset X$.

(b) If $E \subset Y$, and if $E$ intersects only countably many $U \in \mathscr{U}$, then $f^{-1}(E)$ has a countable base.

Proof. Write $\mathscr{U}=\left\{U_{\alpha}: \alpha \in A\right\}$, with $U_{\alpha} \neq U_{\beta}$ if $\alpha \neq \beta$. For all $n \in N$, let $A_{n}$ be the set $A$ with the discrete topology. Let $X$ be the set of all $\alpha=$ $\left(\alpha_{n}\right)$ in $\prod_{n=1}^{\infty} A_{n}$ such that $\left\{U_{\alpha_{n}}: n \in N\right\}$ is a base at some $y_{\alpha} \in Y$. Since $Y$ is Hausdorff, this $y_{\alpha}$ is unique, so we define $f: X \rightarrow Y$ by $f(\alpha)=y_{\alpha}$. All this is standard construction and it is well known-and easy to check-that $f$ is an open map from $X$ onto $Y$ which satisfies (b).

It remains to verify (a). Let $\mathscr{U}_{n} \subset \mathscr{U}(K)$ be as in Lemma 3.3, and pick finite $B_{n} \subset A_{n}$ such that $\mathscr{U}_{n}=\left\{U_{\alpha}: \alpha \in B_{n}\right\}$ for all $n$. Let

$$
C=\left\{\alpha \in \prod_{n=1}^{\infty} B_{n}: \varnothing \neq\left(U_{\alpha_{n+1}} \cap K\right)^{--} \subset U_{\alpha_{n}} \text { for all } n\right\} .
$$

Clearly $C$ is closed in $\prod_{n=1}^{\infty} B_{n}$, and hence compact. If $\alpha \in C$, then $K \cap \bigcap_{n=1}^{\infty} U_{\alpha_{n}} \neq \varnothing$ (since $K$ is compact), so 3.3(a) implies that $\alpha \in X$ and $f(\alpha) \in K$. Hence $C \subset X$ and $f(C) \subset K$. By 3.3(b) we conclude that $f(C)=K$, and that completes the proof.

\section{Examples.}

EXAMPLE 5.1. There exists a cosmic ${ }^{7}$ space $Y$ with the following properties:

(a) $Y$ is an open image of a metric space.

(b) $Y$ is a compact-covering image of a metric space.

(c) $Y$ is not a compact-covering open image of a metric space.

Proof. Let $Y$ be the upper half plane (including the $x$-axis), topologized with the "butterfly topology" of L. F. McAuley. This space is

7 A space is cosmic [18, p. 993] if it is regular and a continuous image of a separable metric space. 
described, for instance, in [18, Example 12.1], where it is observed that it is cosmic and first-countable. Since $Y$ is first-countable, it satisfies (a) by a result of V. I. Ponomarev [22] and S. Hanai [11]. Since $Y$ is cosmic, it has a $G_{\delta}$-diagonal, so every compact subset is metrizable; hence (b) follows from Theorem 1.1. Finally, an observation of C. J. R. Borges [5, Remark 3.3] implies that $Y$ has a compact subset which is not of countable character, so (c) follows from Theorem 1.2. That completes the proof.

According to a theorem of V. V. Filippov [9], every paracompact $p$-space ${ }^{8}$ with a point-countable base is metrizable. Similarly, results of G. Creede [8], R. W. Heath [12] and R. H. Bing [4] imply that every cosmic space with a point-countable base is metrizable. The following two examples show that in neither of these results can the assumption of a point-countable base be weakened to assuming only that every compact subset is metrizable and of countable character.

EXAMPLE 5.2. There exists a (Lindelöf) paracompact $p$-space $Y$ which satisfies the condition of Theorem 1.2 but not that of Theorem 1.3.9

Proof. Let $X=I \times\{0,1\}$ (where $I$ is a closed interval), ordered lexicographically, with the order topology. It is known that $X$ is a compact Hausdorff space, hereditarily separable, and that no uncountable subset of $X$ has a countable base.

Let $E$ be an uncountable subset of $I$, all of whose compact subsets are countable $[14$, p. 514 , Theorem 1]. Let $f: X \rightarrow I$ be the continuous projection, and let $Y=f^{-1}(E)$.

Since $f$ is perfect, so is $f \mid Y$, and hence $Y$ is a paracompact $p$-space. If $K \subset Y$ is compact, then $f(K)$ is compact in $E$ and therefore countable, so $K$ is also countable, and hence $K$ has a countable $Y$-base (since $Y$ is first-countable). Thus, by Lemma 3.1, $Y$ satisfies the condition of Theorem 1.2. Since $Y$ is separable and does not have a countable base, it cannot have a point-countable base. That completes the proof.

EXAMPLE 5.3. There exists a cosmic space $Z$ which satisfies the condition of Theorem 1.2 but not that of Theorem 1.3.10

Proof. Let $Y$ be the upper half plane with the "butterfly topology" which was considered in Example 5.1. This space is cosmic (hence hereditarily separable) and first-countable. Let $Y^{*}=\left\{\left(y_{1}, y_{2}\right) \in Y: y_{2}\right.$ rational $\}$. Define the map $f: Y^{*} \rightarrow R$ by $f\left(y_{1}, y_{2}\right)=y_{1}$. Let $E$ be an uncountable subset

\footnotetext{
${ }^{8}$ A paracompact p-space is a Hausdorff space which admits a perfect map (i.e. a closed map with compact point-inverses) onto a metric space.

${ }^{9}$ This example was obtained independently by F. Siwiec.

${ }^{10}$ Our example is a slight modification of an example given (without proof) by $\mathrm{R}$. W. Heath in [13, Example 4.1]. The modification helps to shorten the proof.
} 
of $R$ all of whose compact subsets are countable [14, p. 514, Theorem 1]. Let $Z=f^{-1}(E)$.

Since $E$ is uncountable, $Z$ does not have a countable base (since any base for $Z$ must contain a butterfly neighborhood of $(x, 0)$ for every $x \in E$ ). The proof that $Z$ satisfies the condition of Theorem 1.2 but not that of Theorem 1.3 now proceeds just like the analogous proof in Example 5.2. That completes our proof.

REMARK 5.4. By the following lemma, the spaces in Examples 5.1-5.3 are neither compact-covering $s$-images nor quotient $s$-images of a metric space.

LEMMA 5.5. If $f: M \rightarrow Y$ is either a quotient s-map or a compact-covering s-map, and if $M$ is metrizable and $Y$ first-countable, then $Y$ has a pointcountable base.

Proof. If $f$ is a quotient $s$-map, then $f$ is bi-quotient by [19, Proposition 3.3(d)], so $Y$ has a point-countable base by a result of V. V. Filippov [10, Theorem 1.1]. If $f$ is a compact-covering $s$-map, then $f$ is a quotient $s$-map, since every compact-covering map whose range is a Hausdorff $k$-space is a quotient map by [18, Lemma 11.2].

Observe that Examples 5.2 and 5.3 could not have been chosen $\sigma$ compact, since Lemma 3.1 implies that a $\sigma$-compact space satisfying the condition of Theorem 1.2 must have a countable base. In contrast to this, the following example is countable.

EXAMPLE 5.6. A countable, regular space $Y$ which is a compactcovering image of a metric space, but which is not a compact-covering $s$-image or a quotient $s$-image of a metric space.

Proof. In [18, Example 12.4], an example is given of a countable, regular space $Y$ which is not an $\boldsymbol{\aleph}_{0}$-space.

Since $Y$ is countable and Hausdorff, every compact subset is metrizable, so $Y$ is a compact-covering image of a metric space by Theorem 1.1.

Since $Y$ is not an $\boldsymbol{K}_{0}$-space, it is neither a compact-covering image nor a quotient image of a separable metric space, by [18, Theorem 11.4 and Corollary 11.5]. But any $s$-map with a countable range must have a separable domain, and hence $Y$ is neither a compact-covering $s$-image nor a quotient $s$-image of a metric space. That completes the proof.

\section{REFERENCES}

1. A. V. Arhangel'skiī, External bases of sets lying in bicompacta, Dokl. Akad. Nauk SSSR 132 (1960), 495-496=Soviet Math. Dokl. 1 (1960), 573-574. MR 22 \#9954.

2. - Factor-mappings of metric spaces, Dokl. Akad. Nauk SSSR 155 (1964), 247-250=Soviet Math. Dokl. 5 (1964), 368-371. MR 29 \#587. 
3. A. V. Arhangel'skii, Bicompact sets and the topology of spaces, Trudy Moskov. Mat. Obšč. 13 (1965), 3-55=Trans. Moscow Math. Soc. 1965, 1-62. MR 33 \#3251.

4. R. H. Bing, Metrization of topological spaces, Canad. J. Math. 3 (1951), 175-186. MR 13, 264.

5. C. J. R. Borges, On stratifiable spaces, Pacific J. Math. 17 (1966), 1-16. MR 32 \#6409.

6. N. Bourbaki, Éléments de mathématique. I: Les structures fondamentales de l'analyse. Fasc. VIII. Livre III: Topologie générale. Chap. 9. Utilization des nombres réels en topologie générale, 2nd ed., Actualités Sci. Indust., no. 1045. Hermann, Paris, 1958; English transl., Hermann, Paris; Addison-Wesley, Reading, Mass., 1966. MR 30 \#3439; MR 34 \#5044b.

7. M. M. Coban, Perfect mappings and spaces of countable type, Vestnik Moskov. Univ. Ser. I Mat. Meh. 22 (1967), no. 6, 87-93. (Russian) MR 36 \#3313.

8. G. D. Creede, Concerning semi-stratifiable spaces, Pacific J. Math. 32 (1970), 47-54. MR 40 \#8006.

9. V. V. Filippov, Feathery paracompacta, Dokl. Akad. Nauk SSSR 178 (1968), 555-558=Soviet Math. Dokl. 9 (1968), 161-164. MR 37 \#3519.

10. - Quotient spaces and multiplicity of a base, Mat. Sb. 80 (122) (1969), 521-532=Math. USSR Sb. 9 (1969), 487-496. MR 41 \#6179.

11. S. Hanai, On open mappings. II, Proc. Japan Acad. 37 (1961), 233-238. MR 23 \#A4116.

12. R. W. Heath, On spaces with point-countable bases, Bull. Acad. Polon. Sci. Sér.

Sci. Math. Astronom. Phys. 13 (1965), 393-395. MR 32 \#4656.

13. - On certain first-countable spaces, Ann. of Math. Studies, no. 60, Princeton Univ. Press, Princeton, N.J., 1965, pp. 103-113.

14. K. Kuratowski, Topologie. Vol. 1, PWN, Warsaw, 1958; English transl., Academic Press, New York; PWN, Warsaw, 1966. MR 19, 873; MR 36 \#840.

15. E. Michael, $A$ theorem on semi-continuous set-valued functions, Duke Math. J. 26 (1959), 647-652. MR 22 \#229.

16. - A class of partially ordered sets, Amer. Math. Monthly 67 (1960), 448-449. MR 22 \#2565.

17. - A note on closed maps and compact sets, Israel J. Math. 2 (1964), 173-176.

MR 31 \#1659.

18. —

19. - Bi-quotient maps and Cartesian products of quotient maps, Ann. Inst. Fourier (Grenoble) 18 (1968), fasc. 2, 287-302. MR 39 \#6277.

20. A. S. Miščenko, Spaces with point-countable base, Dokl. Akad. Nauk SSSR 144 (1962), 985-988=Soviet Math. Dokl. 3 (1962), 855-858. MR 25 \#1537.

21. K. Nagami, Minimal class generated by open compact and perfect mappings (to appear).

22. V. I. Ponomarev, Axioms of countability and continuous mappings, Bull. Acad. Polon. Sci. Sér. Sci. Math. Astronom. Phys. 8 (1960), 127-134. (Russian) MR 22 \#7109.

\footnotetext{
Department of Mathematics, University of Washington, Seattle, Washington 98195
}

Department of Mathematics, Ehime University, Matsuyama, Japan 